\title{
Huge Retroperitoneal Mass: Ginecologic-Type Leiomyoma
}

\section{Rocio Bruballa ${ }^{1 *}$, Ignacio Fuente ${ }^{1,2}$ and Axel Beskow ${ }^{1,2}$}

${ }^{1}$ General Surgery Service Hospital Italiano de Buenos Aires, Juan D. Perón 4190, C1199ABD Buenos Aires, Argentina

${ }^{2}$ Department of Esophageal-Gastro-Duodenal Surgery. Hospital Italiano de Buenos Aires, Juan D. Perón 4190, C1199ABD Buenos Aires, Argentina

${ }^{\star}$ Corresponding author: Dr. Bruballa Rocio, General Surgery Service Hospital Italiano de Buenos Aires, Juan D. Perón 4190, C1199ABD Buenos Aires, Argentina; Tel: (+54) (911) 1536796658; Fax: (+5411) 4959-0200 extn. 4422; E-mail: rocio.bruballa@hospitalitaliano.org.ar

Received: July 01, 2021; Accepted: July 06, 2021; Published: July 07, 2021

\begin{abstract}
Uterine leiomyomas are the most common gynecological tumors in women of reproductive age. However, there are cases of atypical localization, which could represent a diagnosis and treatment challenge. We describe the case of a 54-year-old female patient, with the finding of a large intra-abdominal mass, compatible with gynecological-type leiomyoma, located at the upper retroperitoneum, successfully diagnosed and treated with laparoscopic surgery.
\end{abstract}

Keywords: Retroperitoneal tumors, Ginecologic-type leiomyoma, Surgery, Laparoscopic surgery

\section{Introduction}

Uterine leiomyomas (also called myomata or fibroids) are the most common pelvic neoplasms in women [1,2]. They arise from the smooth muscle cells of the myometrium and extrauterine locations are extremely rare. Although they are histologically benign, in the presence of an atypical presentation, they could mimic malignant tumors at imaging and may become a diagnostic and treatment challenge that will require a multidisciplinary approach $[3,4]$.

The case of a 54-year-old female patient, diagnosed with a large intra-abdominal mass, compatible with gynecological-type leiomyoma, located at the upper retroperitoneum, is presented.

\section{Case Presentation}

A 54-year-old female patient, with a history of hysterectomy for uterine fibroids, was diagnosed with an asymptomatic giant retroperitoneal mass, detected by ultrasound, as part of medical followup. In order to better assess this finding, a Magnetic Resonance Image (MRI) was carried out showing a left retroperitoneal lesion of $142 \times 88$ x $86 \mathrm{~mm}$, of probable mesenchymal origin, causing displacement of the splenic vein, tail of the pancreas, kidney and spleen, with no clear dependence on any organ (Figure 1A and 1B).

The case was discussed on a multidisciplinary committee and based on the size and unknown origin of the lesion, she was considered

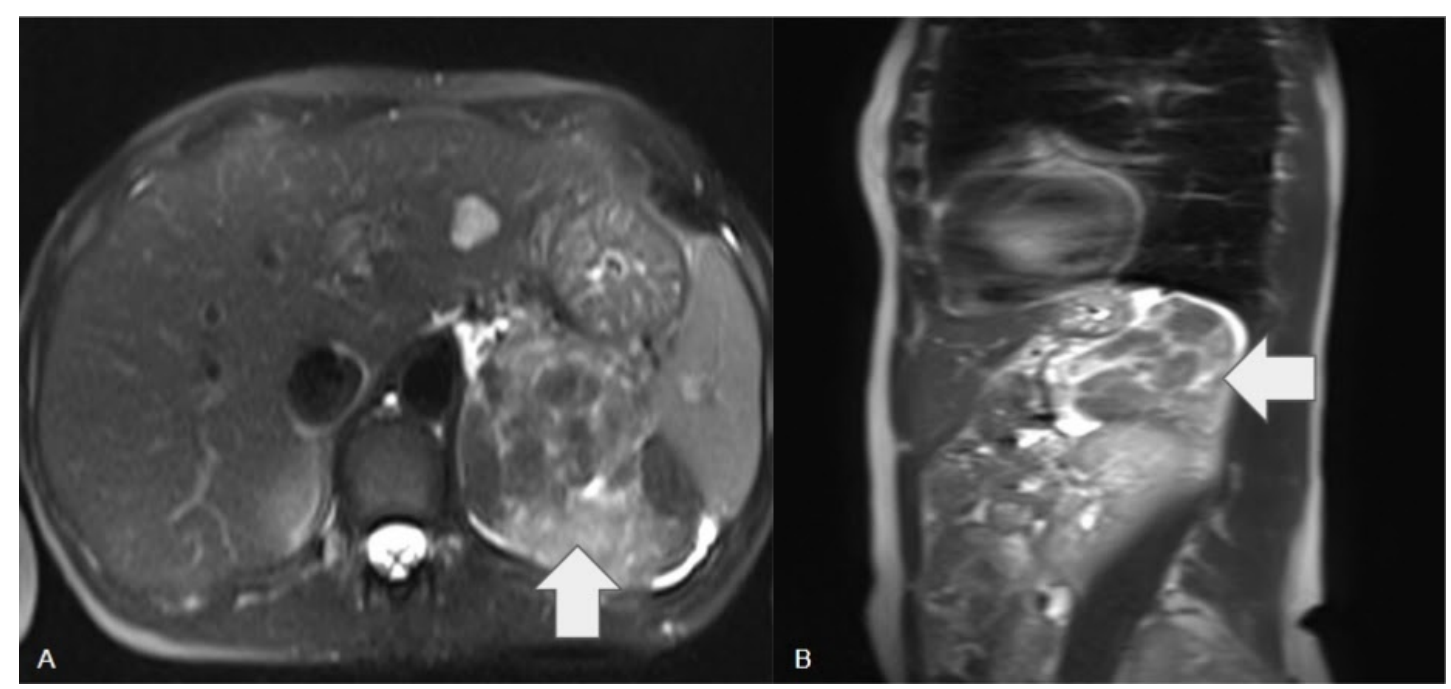

Figure 1: MRI (A: Axial and B: coronal views) showing an heterogeneous retroperitoneal mass of 142 x 88 x $86 \mathrm{~mm}$. 
a candidate for resection. The patient was placed in a lateral decubitus position and a laparoscopic approach was performed. Retroperitoneum was accessed by previously dissecting the sigmoid colon in a lateral to medial fashion. After identification of the mass, a complete resection was performed emphasising not to open the tumor's capsule (Figure 2). The postoperative course was uneventful and the patient was discharged on the third postoperative day. Pathological analysis of the resected specimen revealed a nodular lesion constituted by a proliferation of elongated, fusiform cells of typical muscle appearance without marked mitotic activity ( $<1$ mitosis in $50 \mathrm{HPF}$ ) (Figure $3 \mathrm{~A})$. Hormonal receptors (Estrogen and Progesterone) showed intense and diffuse positivity (Figure 3B). These findings are consistent with gynecologic type retroperitoneal leiomyoma.

\section{Discussion}

Leiomyomas represent the most common gynecologic and uterine neoplasms, diagnosed in up to $70 \%$ of women during their lifetime [5]. They originate primarily from smooth muscle cell proliferation
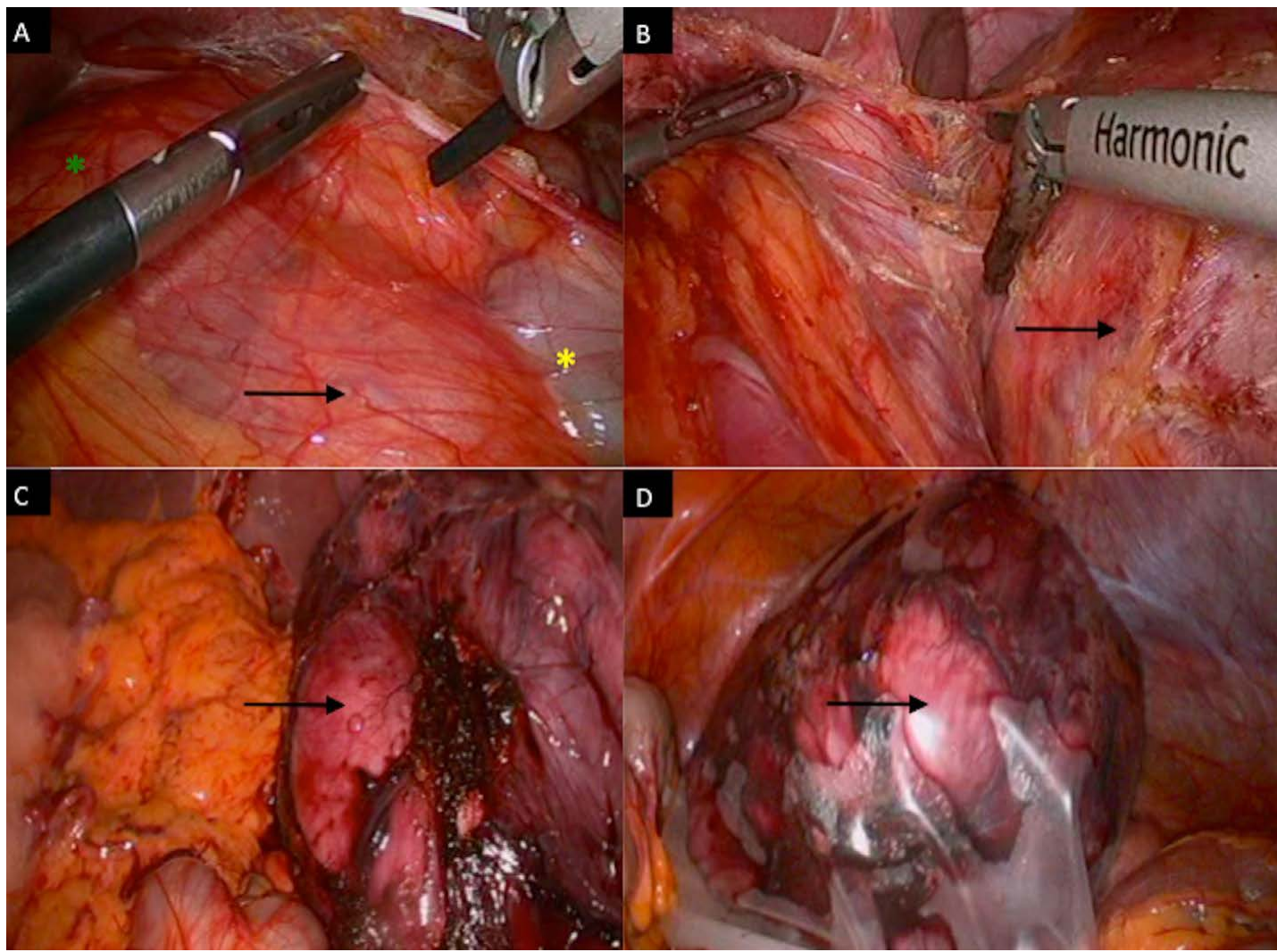

Figure 2: Laparoscopic image showing retroperitoneal mass (black arrow), sigmoid colon (yellow asterisk), left kidney (green asterisk) (A, B and C). After complete dissection, the extraction was performed in a protective bag through a pfannenstiel incision (D).

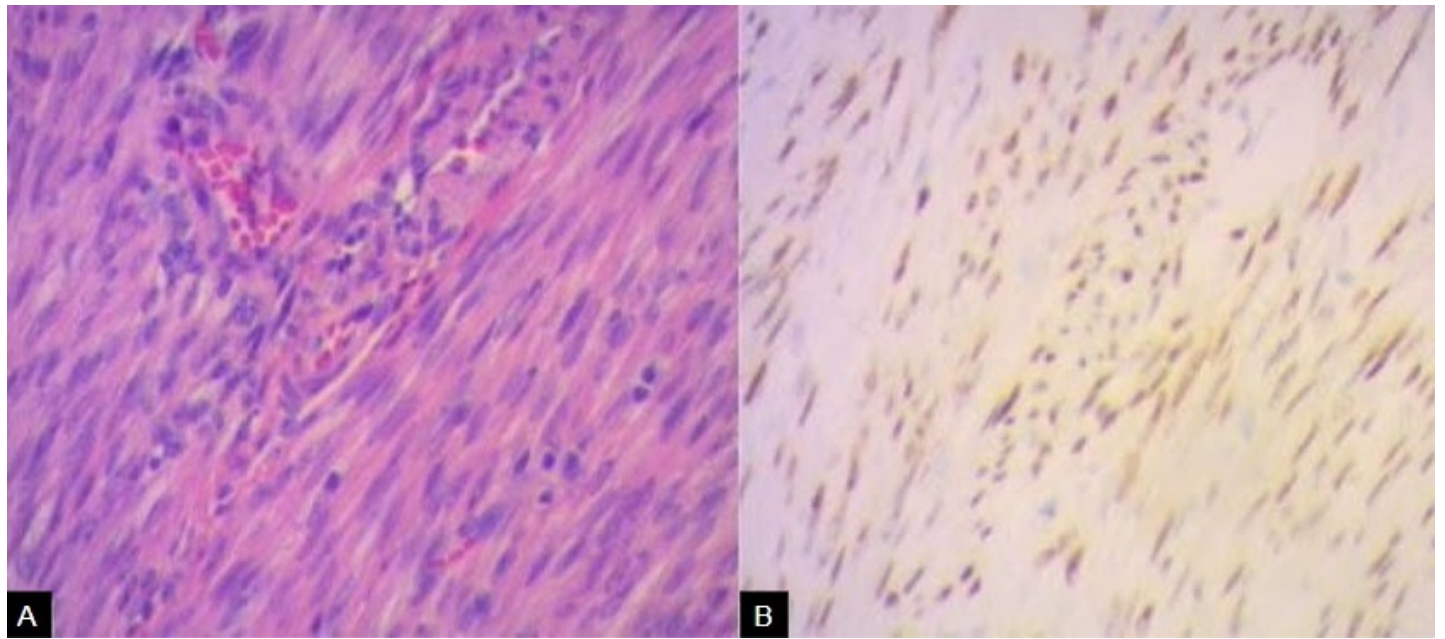

Figure 3: A: Gynecologic-type leiomyoma of retroperitoneum. Hematoxylin-Eosin (H-E) staining sections show intersecting fascicles of slender tapered smooth muscle cells arranged in a whorled pattern separated by well vascularized connective tissue. B: Gynecologic-type leiomyoma of retroperitoneum. Estrogen receptor protein (ER) nuclear staining shows irregular packets and fascicles of spindle cells. 
in the myometrium and extrauterine locations are extremely rare [6]. Although they are histologically benign, extrauterine leiomyomas may mimic malignant tumors at imaging and may become a diagnostic challenge [6,7].

Analyzing the differential diagnoses to be taken into account when facing a retroperitoneal mass, a wide range of tumors can be found, both benign and malignant. Generally, they are divided into solid or cystic, based on the different imaging modalities [8]. In turn, each subgroup is subdivided into neoplastic and non-neoplastic $[9,10]$. The real incidence of each of these pathologies is unknown [11]. However, it has been shown that $80 \%$ of primary retroperitoneal neoplasms are malignant [12]; in fact the retroperitoneal space is the second most frequent location, followed by the lower extremities, where malignant mesenchymal tumors arise. Approximately, one third of retroperitoneal tumors are sarcomas [13]. The most frequent sarcomas are liposarcoma, malignant fibrous histiocytoma, and leiomyosarcoma [14,15]. Given that treatment options vary, it is useful to be able to noninvasively distinguish these masses, this is why preoperative imaging including MRI must be performed [16]. Nevertheless, is a fact, that most of the times it will not be possible to define the tumor's nature [17]. Due to the lack of diagnostic accuracy, using currently available radiologic modalities, prompt surgical intervention will usually be indicated; more if we take into account that these tumors are usually asymptomatic and they may become huge masses before diagnose.

A laparoscopic approach is technically feasible and safe, and should be considered for this cases, given the well described advantages of this approach such as less postoperative pain, rapid recovery, and better cosmetic results $[10,18]$. However, the size and location could potentially be factors to hinder laparoscopic feasibility. If adequate safety margins cannot be ensured, and risk of opening the tumor's capsule is present, an open procedure should be performed [19].

\section{Conclusion}

The relevance of the present case lies in the unusual presentation of a gynecologic type leiomioma as a retroperitoneal mass. As mentioned before, it must be taken into account in the differential diagnosis of retroperitoneal masses, especially when the patient has a history of leiomyoma.

\section{Acknowledgments}

The authors would like to thank the pathology department of the Hospital Italiano de Buenos Aires, for their services.

\section{Conflicts of Interest}

The authors declare not having any conflicts of interest.

\section{Ethical Disclosures}

\section{Protection of Human and Animal Subjects}

The authors declare that no experiments were performed on humans or animals for this study.

\section{Confidentiality of Data}

The authors declare that they have followed the protocols of their work center on the publication of patient data.

\section{Right to Privacy and Informed Consent}

The authors have obtained the written informed consent of the patients or subjects mentioned in the article. The corresponding author is in possession of this document.

\section{References}

1. Stewart EA, Cookson CL, Gandolfo RA, Schulze-Rath R (2017) Epidemiology of uterine fibroids: a systematic review. BJOG: An International Journal of Obstetrics \& Gynaecology 124: 1501-1512. [crossref]

2. Victory R, Romano W, Bennett J, Diamond MP (2015) Uterine leiomyomas: epidemiology, diagnosis, and management. Clinical Gynecology 223-252.

3. Fasih N, Shanbhogue AKP, Macdonald DB, Fraser-Hill MA, Papadatos D, et al (2008) Leiomyomas beyond the uterus: Unusual locations, rare manifestations. Radiographics 28: 1931-1948. [crossref]

4. Poliquin V, Victory R, Vilos GA (2008) Epidemiology, Presentation, and Management of Retroperitoneal Leiomyomata: Systematic Literature Review and Case Report Journal of Minimally Invasive Gynecology 15: 152-160. [crossref]

5. Giuliani E, As-Sanie S, Marsh EE (2020) Epidemiology and management of uterine fibroids. Int. J. Gynaecol. Obstet 149: 3-9. [crossref]

6. Chin H, Ong XH, Yam PKL, Chern BSM (2014) Extrauterine fibroids: a diagnostic challenge and a long-term battle. BMJ Case Rep 2014: 2014204928. [crossref]

7. Takeda T, Asaoka D, Fukumura Y, Watanabe S (2017) Asymptomatic giant retroperitoneal mass detected at a medical checkup. Clin Case Rep 5: 2148-2150. [crossref]

8. Rajiah P, Sinha R, Carlos C, Dubinsky TJ, Bush WHJ, et al. (2011) Imaging of Uncommon Retroperitoneal Masses. RadioGraphics 31: 949-976. [crossref]

9. Osman S, Lehnert BE, Elojeimy S, Cruite I, Mannelli L, et al. (2013) A Comprehensive Review of the Retroperitoneal Anatomy, Neoplasms, and Pattern of Disease Spread. Current Problems in Diagnostic Radiology 42: 191-208. [crossref]

10. Wee-Stekly WW, Mueller MD (2014) Retroperitoneal Tumors in the Pelvis: A Diagnostic Challenge in Gynecology. Frontiers in Surgery 1: 49.

11. Scali EP, Chandler TM, Heffernan EJ, Coyle J, Harris AC, et al. (2015) Primary retroperitoneal masses: what is the differential diagnosis? Abdom Imaging 40: 18871903. [crossref]

12. Neville A, Herts B (2004) CT Characteristics of Primary Retroperitoneal Neoplasms. Critical Reviews in Computed Tomography 45: 247-270. [crossref]

13. Clark MA, Fisher C, Judson I, Meirion Thomas J (2005) Soft-Tissue Sarcomas in Adults. New England Journal of Medicine 353: 701-711.

14. Francis IR (2005) Retroperitoneal sarcomas. Cancer Imaging 5: 89-94.

15. Gupta AK, Cohan RH, Francis IR, Sondak VK, Korobkin M (2000) CT of Recurrent Retroperitoneal Sarcomas. American Journal of Roentgenology 174: 1025-1030. [crossref]

16. Shah JD, Kirshenbaum M, Shah KD (2008) CT Characteristics of Primary Retroperitoneal Tumors And the Importance of Differentiation From Secondary Retroperitoneal Tumors. Contemporary Diagnostic Radiology 31: 1-5.

17. Fasih N, Shanbhogue AKP, Macdonald DB, Fraser-Hill MA, Papadatos D, et al. (2008) Leiomyomas beyond the Uterus: Unusual Locations, Rare Manifestations. RadioGraphics 28: 1931-1948. [crossref]

18. Tsivian M, Ami Sidi A, Tsivian A (2009) Laparoscopic Management of Retroperitoneal Masses: Our Experience and Literature Review. World Journal of Laparoscopic Surgery with DVD 1-5.

19. Cadeddu MO, Mamazza J, Schlachta CM, Seshadri PA, Poulin EC (2001) Laparoscopic Excision of Retroperitoneal Tumors. Surgical Laparoscopy, Endoscopy \& Percutaneous Techniques 11: 144-147. [crossref]

Citation: 